\title{
OPEN ATP amplifies NADPH-dependent and -independent neutrophil extracellular trap formation
}

\author{
Aderonke Sofoluwe $\mathbb{D}^{1,2}$, Marc Bacchetta ${ }^{1,2}$, Mehdi Badaoui ${ }^{1,2}$, Brenda R. Kwak $\mathbb{D}^{3}$ \& \\ Marc Chanson $\mathbb{1}^{1,2^{*}}$
}

Neutrophils are the first immune cells to kill invading microbes at sites of infection using a variety of processes, including the release of proteases, phagocytosis and the production of neutrophil extracellular traps (NETs). NET formation, or NETosis, is a specific and highly efficient process, which is induced by a variety of stimuli leading to expulsion of DNA, proteases and antimicrobial peptides to the extracellular space. However, uncontrolled NETosis may lead to adverse effects and exert tissue damage in pathological conditions. Here, we show that the ATP channel pannexin1 ( $P a n \times 1)$ is functionally expressed by bone marrow-derived neutrophils (BMDNs) of wild-type (WT) mice and that ATP contributes to NETosis induced in vitro by the calcium ionophore A23187 or phorbol 12-myristate 13-acetate (PMA). Interestingly, neutrophils isolated from Panx1 $1^{-1-}$ mice showed reduced and/or delayed induction of NETosis. Brilliant blue FCF dye (BB-FCF), a Panx1 channel inhibitor, decreased NETosis in wild-type neutrophils to the extent observed in ${\mathrm{Pan} \times 1^{-1-}}^{-1}$ neutrophils. Thus, we demonstrate that ATP and Panx1 channels contribute to NETosis and may represent a therapeutic target.

Neutrophils mature in the bone marrow, where they synthesise and package enzymes and antimicrobial proteins into secretory granules that are rapidly released at sites of infection and inflammation ${ }^{1}$. Among several antimicrobial defence mechanisms, the externalisation of decondensed nuclear chromatin was first described to capture and trap invading pathogens and facilitate their killing ${ }^{2}$. The formation of neutrophil extracellular trap (NET), also known as NETosis, is triggered by pathogen-associated virulence factors and pro-inflammatory stimuli ${ }^{2-4}$. Recent studies have provided insights into the signalling pathways and the characteristics of different forms of NETosis triggered by different stimuli. Suicidal NETosis is commonly observed in inflammatory diseases such as rheumatoid arthritis, lupus and vasculitis in which autoantibodies target released NETs ${ }^{5}$. Suicidal NETosis is a slow process that eventually leads to cell death through the generation of free reactive oxygen species (ROS) that activate peptidyl arginine deiminase type IV (PAD4), myeloperoxidase-dependent nuclear envelope degradation and subsequent release of nuclear and cellular contents $s^{5,6}$. NETs were originally observed in response to the protein kinase $\mathrm{C}$ activator phorbol 12-myristate 13-acetate (PMA), which induces NETosis through the ROS generating NADPH oxidase complex that contributes to the disruption of the extracellular membrane $e^{6,7}$. In contrast, vital NETosis is a faster and robust, NADPH-independent, process dominated by a rise in intracellular calcium concentration and often observed during infection ${ }^{8,9}$. A third type of very fast NETosis is characterised by the release of mitochondrial DNA through the recognition of granulocyte-macrophage colony-stimulating factor or lipopolysaccharide ${ }^{10}$. It is believed that end-stage of NETosis requires PAD4 citrullination of histone $\mathrm{H} 3$, which could play a major role in formation and/or function of NETs ${ }^{11,12}$. Recently, a biophysical model for NETosis was proposed whereby, independent of the stimulus, entropic swelling of the chromatin represents the main physical force leading to rupture of the nuclear envelope and the plasma cell membrane ${ }^{13}$. This is important because the model predicts that a point of no return is reached once NETosis is initiated.

Through multiple trigger mechanisms, excessive NETosis may exert tissue damage and enhance the severity of pathological conditions ${ }^{12}$. For instance, NETs are detected in multiple organs in sepsis, and may enhance inflammation and viscosity of sputum in cystic fibrosis ${ }^{14,15}$. NETs were shown to be present in the thrombus in deep vein thrombosis and to be targets of autoantibodies in autoimmune diseases like rheumatoid arthritis and systemic

\footnotetext{
${ }^{1}$ Department of Paediatrics, Gynaecology \& Obstetrics, Geneva University Hospitals, Geneva, Switzerland. ${ }^{2}$ Department of Cell Physiology \& Metabolism, University of Geneva, Faculty of medicine, Geneva, Switzerland. ${ }^{3}$ Department of Pathology \& Immunology, University of Geneva, Faculty of medicine, Geneva, Switzerland. *email: Marc.Chanson@unige.ch
} 
lupus erythematosus ${ }^{3,5}$. Thus, there is a need to identify NET inhibitors and new therapeutic targets to prevent worsening conditions in NETs-related diseases.

Accumulating evidence shows that innate immune responses are associated with extracellular nucleotides, particularly adenosine $5^{\prime}$-triphosphate (ATP). ATP acts as an extracellular signalling molecule that can modulate cellular processes through activation of specific cell membrane purinergic receptors. Under inflammatory conditions, ATP is present in the extracellular space due to its release from the cell cytoplasm. Among other mechanisms, pannexin-made membrane channel might be essential for inside out signalling by releasing ATP to the extracellular space ${ }^{16}$. Pannexin (Panx) family consists of three isoforms (Panx1, Panx2 and Panx3), Panx1 being ubiquitously expressed ${ }^{17,18}$. Neutrophils were found to release ATP through Panx1 channels, which in turn activates purinergic receptors in a paracrine manner ${ }^{19,20}$. In this study, we questioned whether ATP and Panx1 contributed to NETosis using wild-type (WT) and $\mathrm{Panx}^{-1-}$ murine neutrophils activated with the calcium ionophore A23187 or PMA. We report here that extracellular ATP amplifies NETosis through purinergic stimulation. We also show that NETosis is reduced in Pan $x 1^{-1-}$ neutrophils and that pharmacological inhibition of Panx1 slowed NETosis triggered by A23187 and PMA.

\section{Results}

NET formation induced by A23187 and PMA. A23187 and PMA are commonly used to trigger vital (NADPH-independent) and suicidal (NADPH-dependent) NETosis. Formation of NET was first verified by immunostaining of citrullinated histones in WT bone marrow derived neutrophils (BMDNs) exposed to A23187 or PMA for $2 \mathrm{~h}$ or untreated. As illustrated in Fig. 1A and Supplemental Fig. 1, histone citrullination was stronger in A23187 stimulated neutrophils when compared to PMA stimulated cells, which is consistent with the fast and robust response evoked by the calcium ionophore ${ }^{21}$.

NET formation can also be monitored as a function of time by using the cell impermeable SYTOX green dye, which binds to extracellular DNA. An example of NET formation by time-lapse imaging in a global population of stimulated BMDNs is shown in Video 1 (Supplemental Video 1). To provide quantitative and temporal analysis of NETosis, SYTOX green dye fluorescence over time was quantified using a plate reader. Typically, increase in SYTOX green fluorescence followed a sigmoidal curve; A23187 induced rapid and robust extracellular DNA release, reaching a plateau within 3-4 h (Fig. 1B; black curve). NET formation induced by PMA was slower and reached maximal extracellular DNA after 4-6h (Fig. 1C; black curve). As expected, BMDN incubation with the antioxidant $\mathrm{N}$-acetyl cysteine (NAC) strongly reduced the induction of NADPH-dependent NETosis while it was inefficient in modulating NADPH-independent NETosis (Fig. 1B,C; grey curves), confirming the specificity of the signalling induced in neutrophils by A23187 and PMA.

Extracellular ATP contributes to NETosis. To evaluate the contribution of ATP and purinergic signalling to NETosis, neutrophils were stimulated with A23187 or PMA in the presence of drugs that would reduce ATP degradation, such as an inhibitor of the surface ecto-enzyme CD39 (ARL67156), or drugs that would inhibit activation of purinergic receptors. As shown in Fig. 2A,B, PMA-induced, but not A23187-induced, NETosis was enhanced in the presence of ARL67156. NADPH-independent NETosis may not be enhanced by ARL67156 due to the already maximal effect caused by the calcium ionophore. In agreement, we found that a P2 $\mathrm{Y}_{2}$ receptor antagonist (AR-C 118925XX) decreased NET formation induced by A23187 and PMA (Fig. 2C,D). These results were corroborated by treating cells with apyrase, an ATP scavenger, which decreased NETosis triggered by A23187 or PMA (Fig. 2E,F). Thus, extracellular ATP may act in an autocrine and/or paracrine manner on purinergic signalling to modulate NETosis.

We next investigated whether ATP alone triggered NETosis. However, neither $50 \mu \mathrm{M}$ nor $500 \mu \mathrm{M}$ of extracellular ATP enhanced SYTOX fluorescence above that of controls (Fig. 3A,B). In addition, combining ATP and A23187 did not modify the time course or the amplitude of NETosis induced by A23187 alone (Fig. 3C,D), confirming that NETosis induced by the calcium ionophore was already maximal. Interestingly, we observed that adding ATP to PMA stimulation markedly enhanced NETosis, following a faster and more pronounced S-shaped kinetic similar to that observed for A23187 stimulation (Fig. 3E,F). Together, the data suggest that extracellular ATP is a modulator of NETosis, the rate and amplitude of which depend of the triggers used and of the purinergic signalling activated.

Panx1 is functionally expressed in neutrophils. Panx1, which forms ATP channels in neutrophils ${ }^{19,20}$, could contribute to ATP release during NETosis. Immunostaining of BMDNs confirmed Panx1 protein expression; Panx1 was mostly detected at the neutrophil membrane (Fig. 4A and Supplemental Fig. 2A). In contrast, Panx1 protein were absent in neutrophils isolated from $P a n x 1^{-1-}$ mice (Fig. 4A). This was also confirmed at the mRNA (Fig. 4B) and at the protein (Supplemental Fig. 2B) levels. To exclude compensatory upregulation of other pannexin family members in Panx $1^{-l-} \mathrm{BMDNs}^{22}$, we verified for Panx2 and Panx3 transcripts, which were not detected in both WT and Panx1 $1^{-1-}$ BMDNs (Fig. 4B). Connexin43 (Cx43) transcripts levels were very low and similar in both genotypes (Fig. 4B), excluding secondary effects from $\mathrm{Cx} 43$, which has been shown to form ATP releasing hemichannels in various cell types ${ }^{20,23}$. Panx1 function was first evaluated in WT and Panx $1^{-1-}$ BMDNs by measurement of ATP release using hypo-osmotic shock (Fig. 4C). Within 5 min after stimulation, there was a marked increase in ATP release in WT neutrophils compared to Pan $x 1^{-1-}$ neutrophils. Interestingly, A23187 or PMA stimulations did not elicit ATP release (Fig. 4C).

YO-PRO-1 dye uptake is another approach to monitor Panx1 function. Global measurement of YO-PRO-1 dye uptake in the overall neutrophil population was detected using a plate reader (Fig. 4D). BMDNs were first superfused with a control solution to acquire baseline fluorescence and YO-PRO-1 dye was then added to the medium. As a trigger to maximally open Panx1 channels, neutrophils were subsequently superfused by a hypotonic solution containing the same concentration of YO-PRO-1 dye. Increases in fluorescent signal were 
A
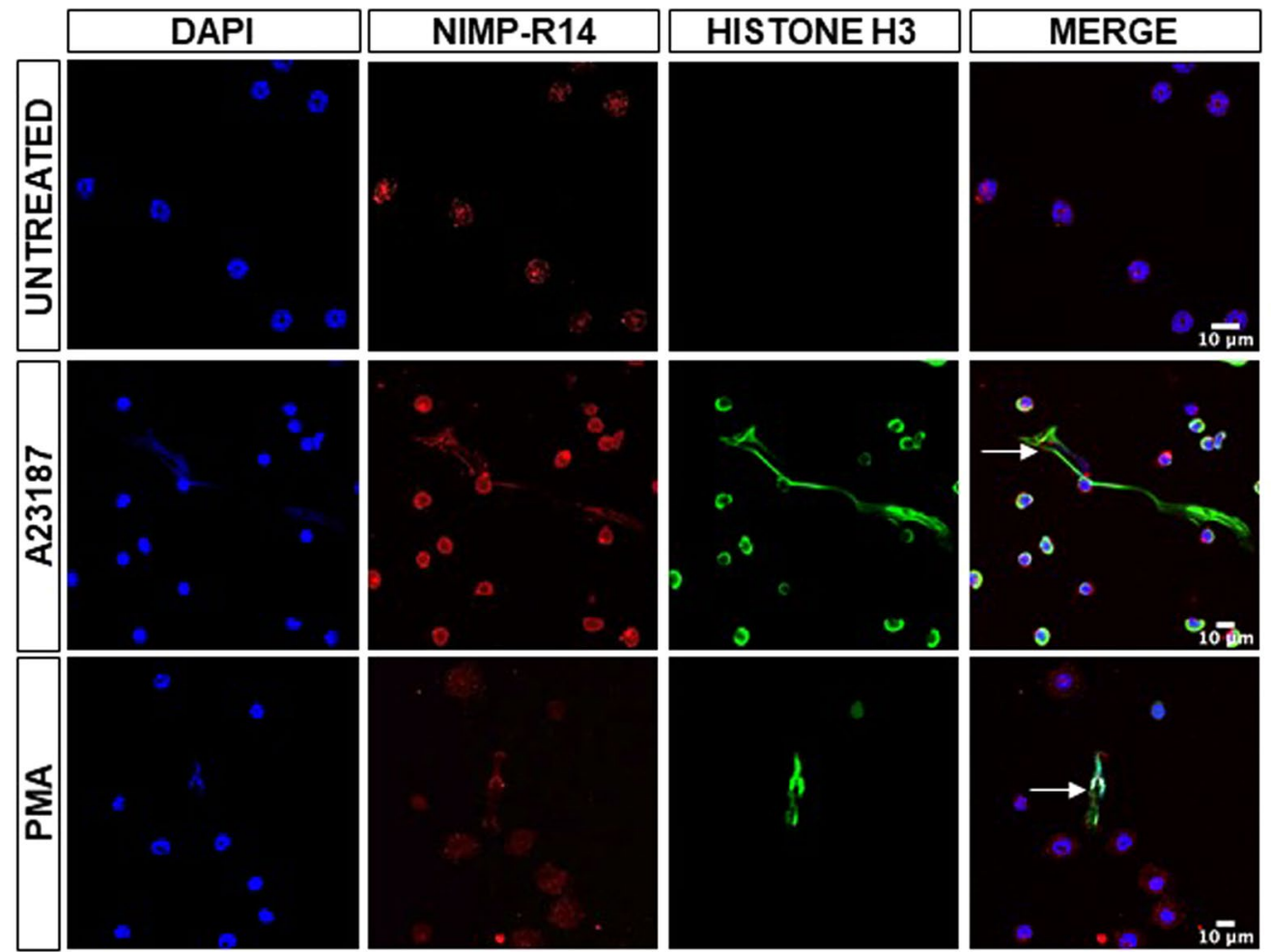

B

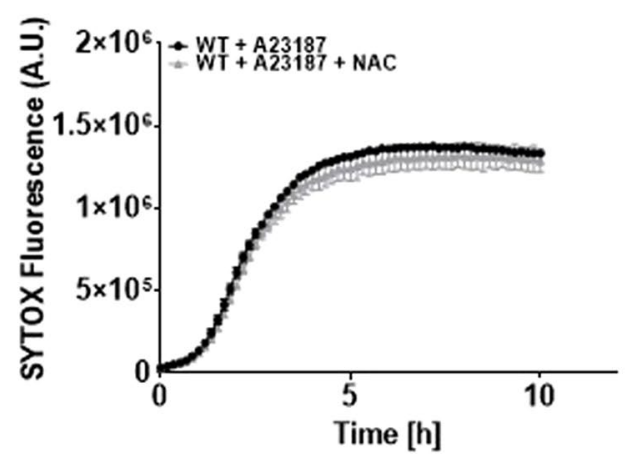

C

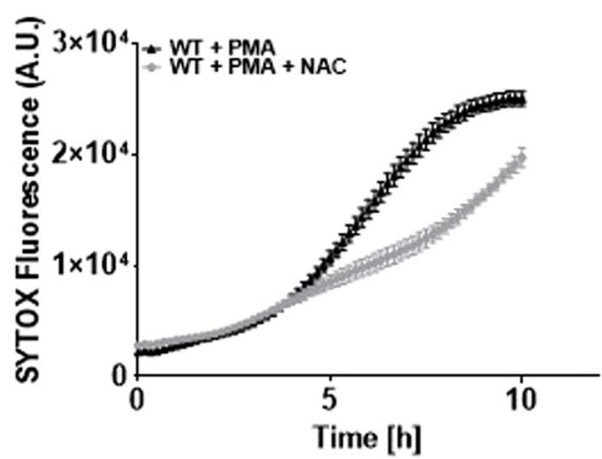

Figure 1. Qualitative and quantitative assessment of NETosis induced with NADPH-dependent and -independent stimuli. (A) Immunofluorescence staining of NETs after $2 \mathrm{~h}$ stimulation revealed histone $\mathrm{H} 3$ (histone citrullination) in green. Neutrophils were stained with the specific marker NIMP-R14 (in red) and nuclei with DAPI (blue). Untreated BMDNs (upper panels), BMDNs stimulated with $1 \mu$ M A23187 (middle panel) and with $50 \mathrm{nM}$ PMA (bottom panel) are shown. White arrows indicate neutrophils with released extracellular traps. $(\mathbf{B}, \mathbf{C})$ Kinetics of SYTOX green fluorescence detection of NETosis in response to $1 \mu \mathrm{M}$ A23187 (B) and $50 \mathrm{nM}$ PMA (C), in the absence or presence of $10 \mathrm{mM} \mathrm{N}$-acetyl-cysteine (NAC). NAC was used to inhibit reactive oxygen species.

observed, the YO-PRO-1 fluorescence being markedly enhanced in WT as compared to Pan $x 1^{-1-}$ neutrophils (Fig. 4D; black vs. grey curves). Importantly, YO-PRO-1 uptake in WT neutrophils decreased back to the level observed for Panx $1^{-I}$ BMDNs in the presence of the Panx1 inhibitors BB-FCF or spironolactone (Fig. 4D; black $v s$. blue and red curves, respectively). Quantification of the overall neutrophil population confirmed the reduction of YO-PRO-1 uptake in Panx $1^{-1-}$ neutrophils and in the presence of Panx1 inhibitors (Fig. 4E). These results indicate that Panx 1 is functionally expressed in BMDNs.

NETosis is delayed in Panx1-I- neutrophils and by Panx1 channel inhibition. To determine if Panx1 channels contribute to NETosis, we compared the kinetics of extracellular DNA detection between WT and Pan $x 1^{-1-}$ BMDNs exposed to A23187 (Fig. 5A) or PMA (Fig. 5B). For both stimuli, the kinetics of NETosis were slowed down in Pan $x 1^{-1-}$ neutrophils. Using linear regression analysis, traces were fit to a sigmoidal curve and we obtained values for maximal response Max (Fig. 5C,D), Hill slope (Fig. 5E,F) and time to half maximal response $\mathrm{ET}_{50}$ (Fig. $5 \mathrm{G}, \mathrm{H}$ ) parameters. Violin plots of the distribution of normalised values are shown. For each experiment, the parameters obtained in $\mathrm{Panx}^{-1-}$ BMDNs were normalised to that measured in WT neutrophils. 
A

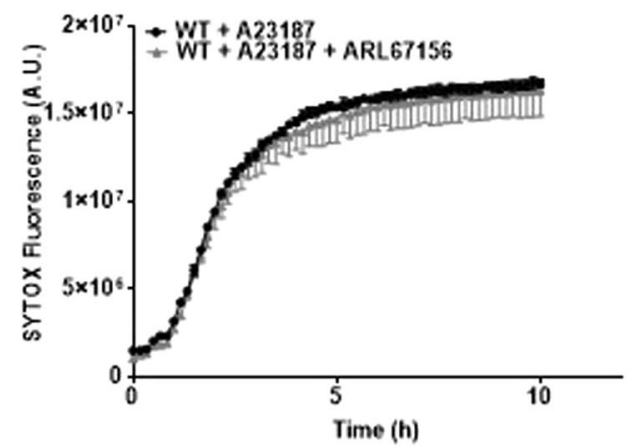

C

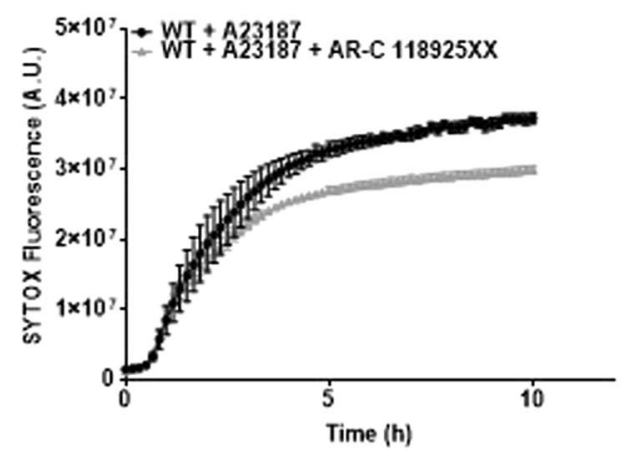

E

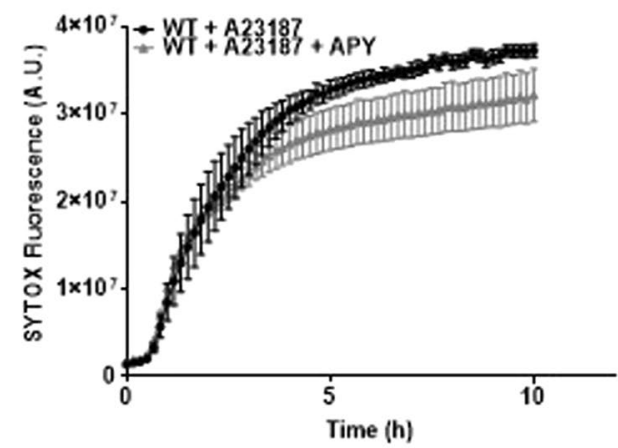

B

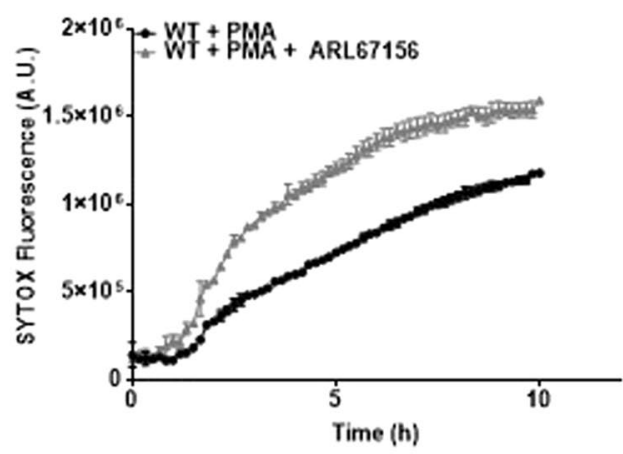

D

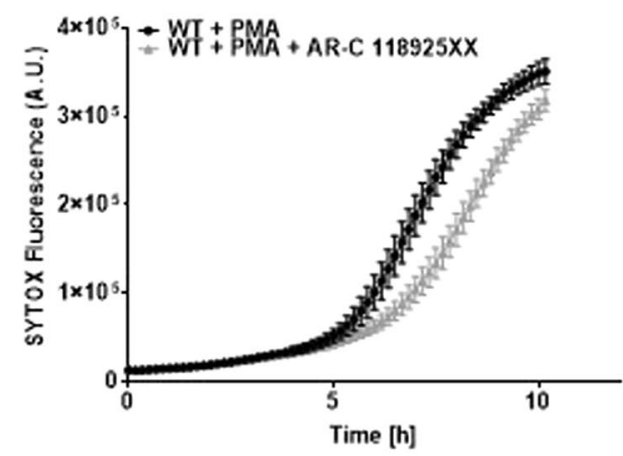

$\mathbf{F}$

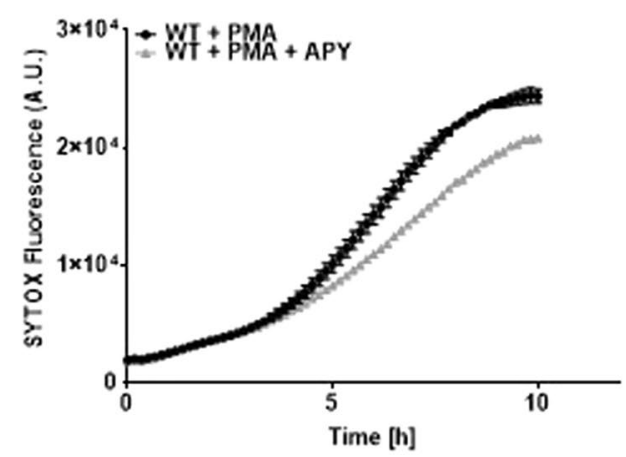

Figure 2. Modulation of NETosis by extracellular ATP. (A,B) Kinetics of ecto-ATPase inhibition using ARL67156 $(100 \mu \mathrm{M})$ in A23187 and PMA stimulation respectively. (C,D) Kinetics of $\mathrm{P}_{2} \mathrm{Y}_{2}$ receptor signalling inhibition using $\mathrm{P}_{2} \mathrm{Y}_{2}$ receptor antagonist AR-C 118925XX (500 nM) in A23187 and PMA stimulation respectively. (E,F) Kinetics of ATP scavenging using $50 \mathrm{U} / \mathrm{ml}$ apyrase (APY) in A23187 and PMA stimulation respectively. Note in $B$ that the initial phase is not shown. $N=3, n=6$.

Although no difference was observed for the Hill slope parameters, Max was decreased in Panx $1^{-1-}$ neutrophils

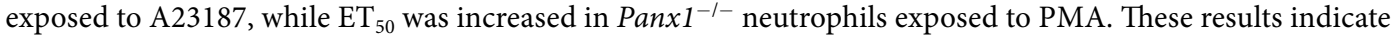
that Panx1 contributes to NET formation and modulates the kinetic of NETosis according to the trigger used.

BB-FCF has been shown to inhibit Panx1 channels in various cell types ${ }^{24,25}$, including neutrophils (Fig. 4D,E). Therefore, we evaluated the ability of the inhibitor to modulate the kinetic of NETosis in response to A23187 and PMA. We observed a delay in the kinetics in BB-FCF-treated WT neutrophils, which was comparable to the behaviour of Panx1 $1^{-1-}$ BMDNs (Fig. 6A,C). After $6 \mathrm{~h}$, comparison of the SYTOX fluorescence measurements showed significant reduction WT neutrophils exposed to BB-FCF (Fig. 6B). We observed similar effects in BB-FCF-treated WT neutrophils with PMA stimulation (Fig. 6D). These results show that Panx1 inhibitor BB-FCF reduced NETosis in WT BMDNs stimulated with both A23187 and PMA.

\section{Discussion}

This study provides evidence that Panxl and extracellular ATP, which is often elevated during inflammation, contribute to NETosis. Since the discovery of $\mathrm{NETs}^{2}$, NETosis is considered as a causal factor in the severity of various diseases. Whereas several intracellular signalling cascades have been identified, NETosis is clearly distinct from other processes causing cell death like apoptosis, necrosis and necroptosis ${ }^{12}$. Importantly, the initial phase of the process is associated with morphological changes and modifications of histones that prime the neutrophils for later mechanical changes. At this point, chromatin swelling is engaged leading to the irreversible rupture of the nuclear envelope and then the plasma cell membrane ${ }^{13}$. 
A

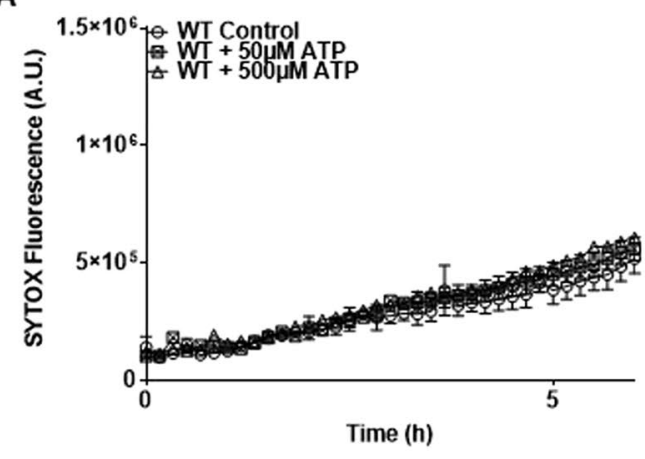

C

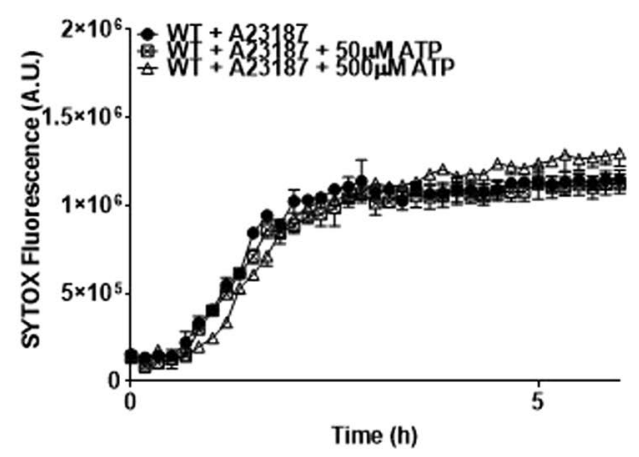

E

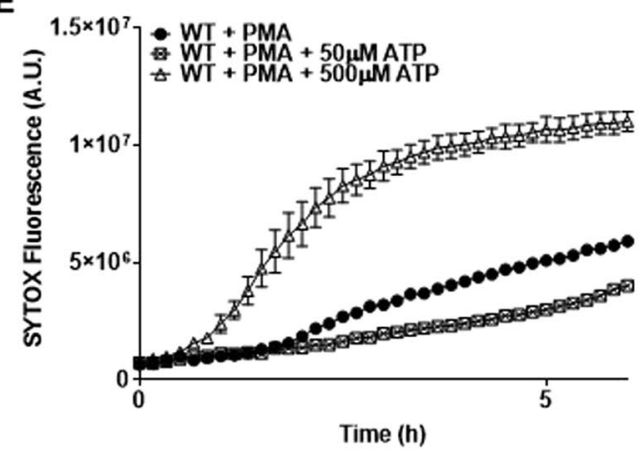

B

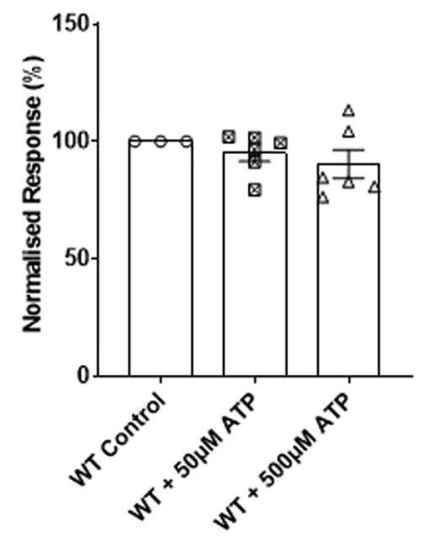

D

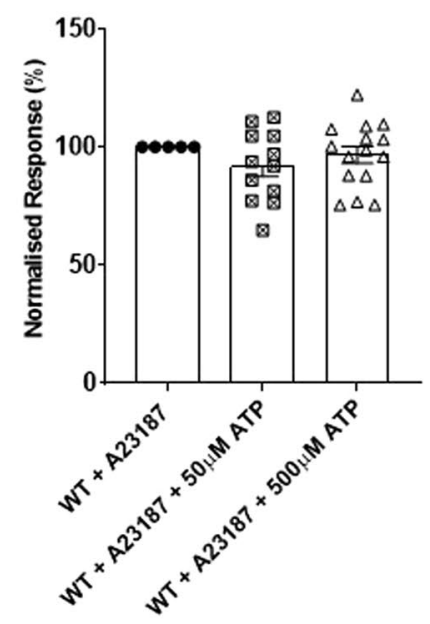

F

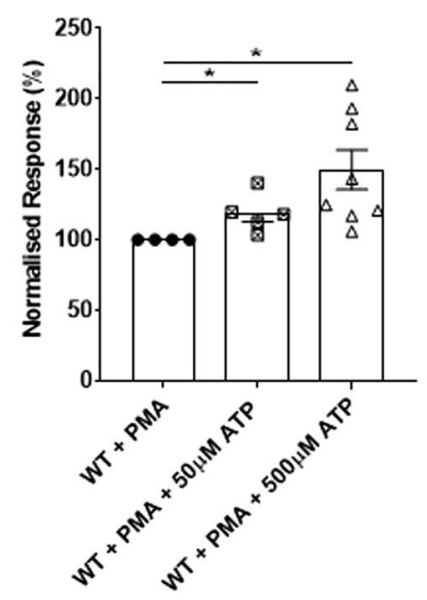

Figure 3. Role of ATP in the modulation of NETosis. (A) Kinetics of ATP stimulation $(50 \mu \mathrm{M}$ and $500 \mu \mathrm{M})$ in WT neutrophils at $6 \mathrm{~h}$. (B) Quantification of ATP stimulation in WT neutrophils at $6 \mathrm{~h}$. (C,E) Kinetics of ATP stimulation $(50 \mu \mathrm{M}$ and $500 \mu \mathrm{M})$ with A23187 and PMA stimulation respectively. (D,F) Quantification of response to ATP stimulation $(50 \mu \mathrm{M}$ and $500 \mu \mathrm{M})$ with A23187 and PMA stimulation respectively. $(\mathrm{N} \geq 3)$. ${ }^{*} \mathrm{P}<0.05$.

A23187 and PMA are commonly used inducers of suicidal and vital NETosis. We confirmed the different dependence of these stimuli to NADPH oxidase and in the level of histone citrullination ${ }^{5,21}$. As expected, after several hours, both stimuli induced the release of DNA to the extracellular space that could be monitored by the use of the cell impermeable dye SYTOX green. The increase in SYTOX green fluorescence measured on a global 
A
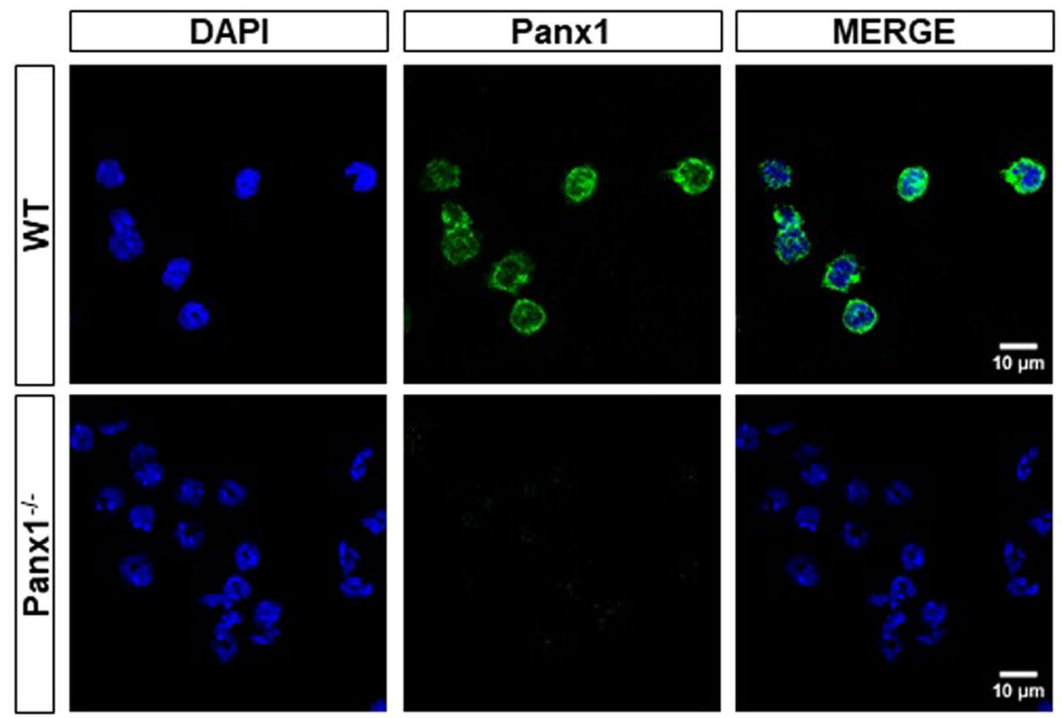

B
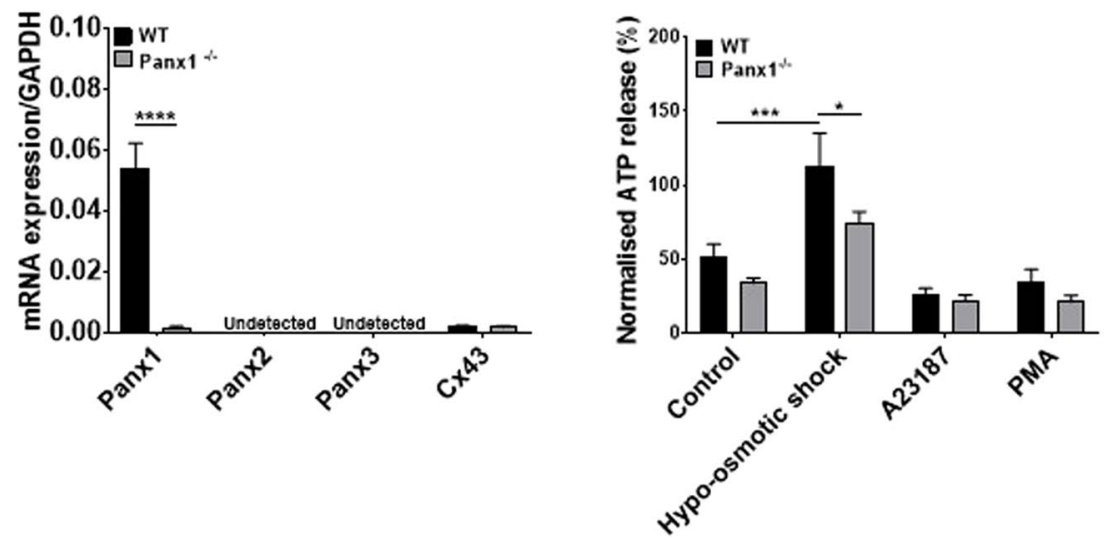

D

E
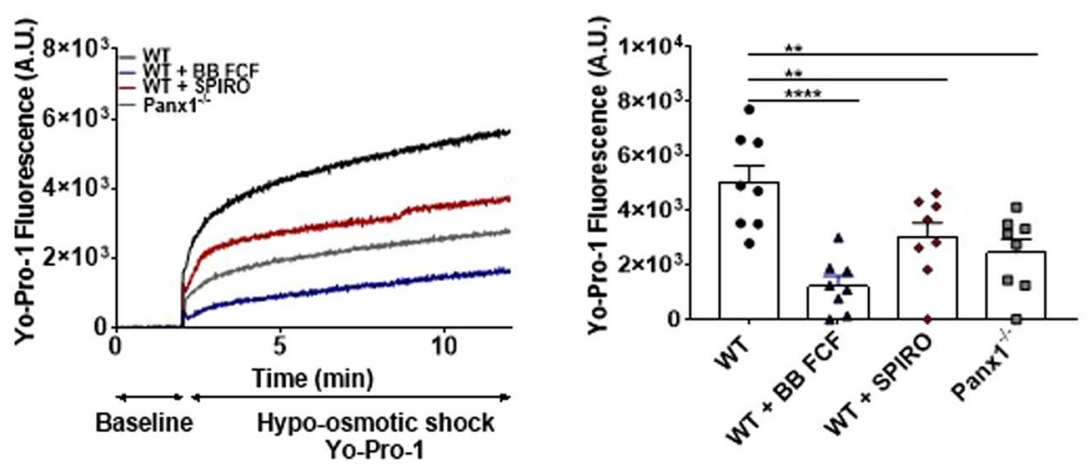

Figure 4. Panx1 expression and function in BMDNs. (A) Immunofluorescence of Panx1 (green), nuclei staining with DAPI (blue) in WT and Pan $x 1^{-1-}$ BMDNs respectively. (B) mRNA expression levels of Panx1, 2, 3 and Cx43 in WT and Panx $1^{-1-}$ BMDNs measured by qPCR $(\mathrm{N}=3, \mathrm{n}>5)$. (C) Quantification of ATP release in WT and Panx1 $1^{-1-}$ BMDNs with hypo-osmotic shock, A23187 and PMA stimulation at 5 minutes $(\mathrm{N}=3, \mathrm{n}=6)$. (D) Representative kinetic of YO-PRO-1 dye uptake in the absence or presence of the Panx1 inhibitors BB-FCF $\left(1 \mu \mathrm{M}\right.$, blue) or spironolactone $\left(100 \mu \mathrm{M}\right.$, red) for WT BMDNs (black) as compared to Pan $x 1^{-1-}$ BMDNs (grey). (E) Quantification of dye uptake in BMDNs $(\mathrm{N}=4) .{ }^{* *} \mathrm{P}<0.01$; ${ }^{* * * *} \mathrm{P}<0.0001$.

population of stimulated BMDNs followed a sigmoidal curve. Consistent with video-microscopy recordings, the sigmoidal curve resulted from an increasing number of neutrophils undergoing NETosis with time. Panx $1^{-1-}$ neutrophils were less sensitive to both A23187- and PMA-induced NETosis, yet different characteristics were 
A

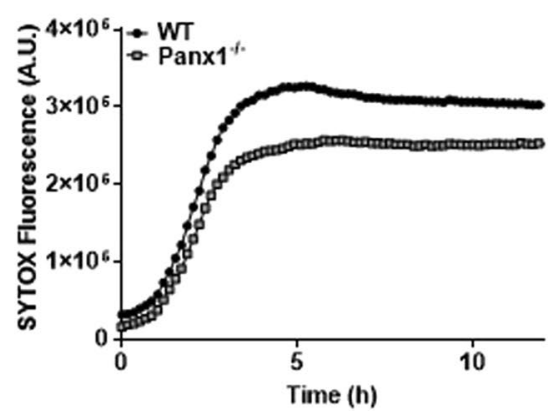

B

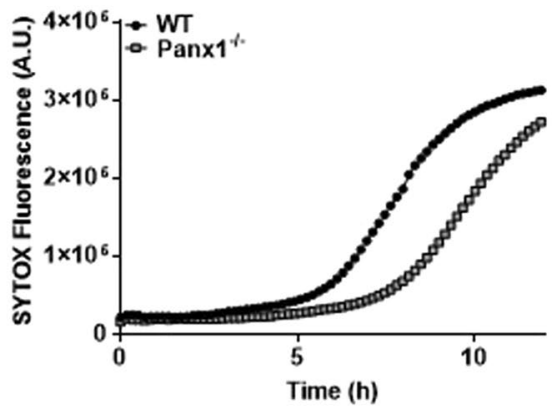

C

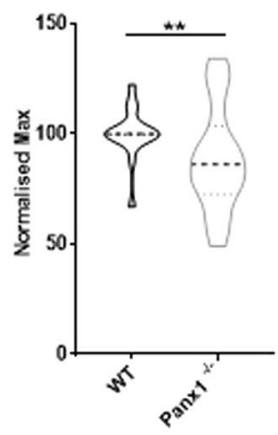

D

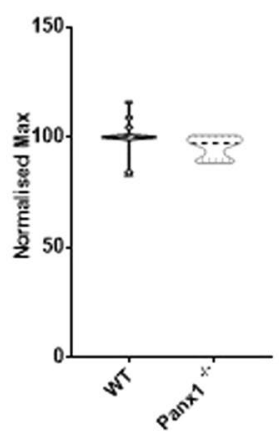

$E$

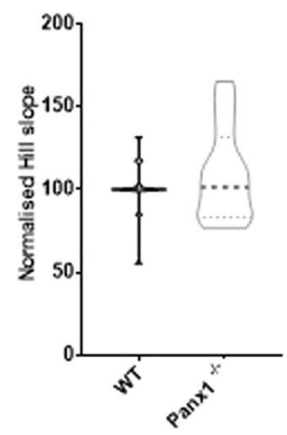

$\mathbf{F}$

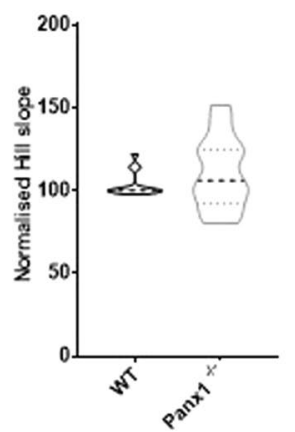

G

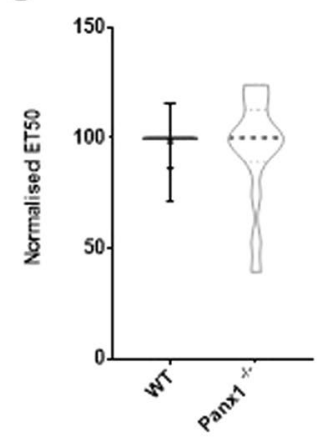

H

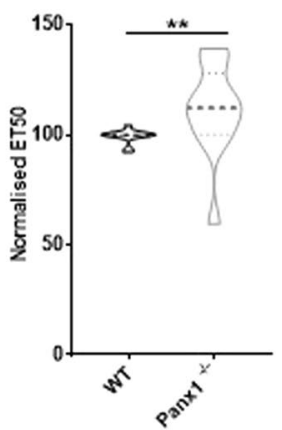

Figure 5. NADPH-dependent and independent NETosis in Panx1 $1^{-1-}$ BMDNs. (A) Representative kinetic of A23187 $(1 \mu \mathrm{M})$ stimulation in WT and Panx $1^{-1-}$ neutrophils with SYTOX fluorescence over time. (B) Representative kinetic of PMA (50 nM) stimulation of WT and Panx1 $1^{-1-}$ neutrophils with SYTOX fluorescence over time. (C,D) Quantification of Maximal (Max) values in A23187 and PMA stimulations respectively $(\mathrm{n}=13)$. (E,F) Quantification of Hill Slope indicating rate of reaction in A23187 and PMA stimulations respectively $(\mathrm{n}=13)$. $(\mathbf{G}, \mathbf{H})$ Quantification of half-maximal time $\left(\mathrm{ET}_{50}\right)$ in A23187 and PMA stimulations respectively.

observed. The number of Panx1 $1^{-1-}$ BMDNs undergoing fast DNA release was reduced in response to A23187. However, DNA release was occurring with slower kinetics in Panx $1^{-1-}$ than in WT neutrophils in response to PMA. Our data also shows decreased ATP release in Pan $x 1^{-1-}$ neutrophils. Together, the results indicate that Panx1 accelerates the stimuli-triggered initial phase that leads to chromatin swelling, possibly by releasing ATP to the extracellular environment.

Purinergic regulation of neutrophil function is well established ${ }^{19,20}$. Extracellular ATP and its hydrolysed derivatives (ADP, AMP and adenosine) are critical signal transduction molecules, the balance of which is regulated by surface ectoenzymes. Thus, ATP and ADP are converted to AMP by ecto-apyrase CD39, which is highly expressed in neutrophils ${ }^{26}$. These nucleotides and nucleosides are ligands to $\mathrm{P} 2$ and $\mathrm{P} 1$ receptors respectively, which convert extracellular purine concentration into intracellular signals that modulate neutrophil immune response. Interestingly, one study reported that $\mathrm{P} 1$ receptor signalling could decrease NETosis induced by Klebsiella pneumonia ${ }^{27}$. The actual concentration of ATP close to the BMDN cell surface, which will vary with time, activity of ectoenzymes and number of activated purinergic receptors, is unknown. Nonetheless, we found that blocking $\mathrm{P}_{2} \mathrm{Y}_{2}$ receptors or scavenging ATP decreased NET formation, pointing to the P2 receptors as a major amplifier of NETosis. Extracellular ATP alone was not able to trigger NETosis, but enhanced NET formation evoked by PMA with kinetics similar to that obtained with A23187 stimulation. $\mathrm{P}_{2} \mathrm{Y}_{2}$ signalling has been previously involved in NET formation during acute phase of cattle neosporosis ${ }^{28}$. Also in line with these data is the report that uridine diphosphate (UDP), which is the natural ligand to $\mathrm{P}_{2} \mathrm{Y}_{6}$ receptor, fails to initiate NET formation in human neutrophils but UDP-mediated $\mathrm{P}_{2} \mathrm{Y}_{6}$ signalling is involved in the monosodium urate crystal-induced formation of NETs ${ }^{29}$. Together, these observations suggests that elevation of intracellular calcium concentration secondary to purinergic receptor activation plays a pivotal role in regulating the velocity of the NET response ${ }^{21}$.

Several mechanisms for ATP release from various cell types have been proposed, including vesicle exocytosis and channel-dependent transport among which connexons (made of connexins) and pannexons (made of pannexins) are extensively studied ${ }^{30}$. The release of ATP through Cx43-made connexons and Panx1 channels was previously reported in neutrophils in response to $\mathrm{N}$-formylmethionyl-leucyl-phenylalanine, lipopolysaccharide or hypertonic saline ${ }^{31,32}$. Although Panx1 mRNA was detected in WT neutrophils, the levels of Panx2, Panx3 and Cx43 mRNA were negligible in both WT and Pan $x 1^{-1-}$ BMDNs, excluding them from a possible compensatory mechanism. The molecular mechanism that opens Panx1 channels in neutrophils exposed to A23187 or PMA have not been examined here. It is worth noting that many cellular changes induced by A23187 and PMA during 
A

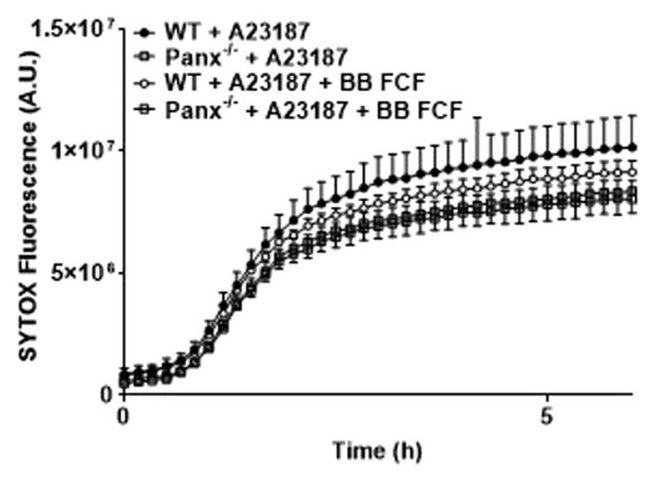

C

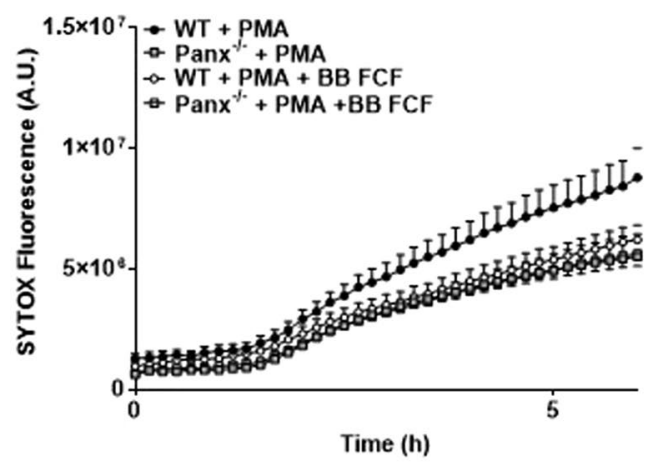

B

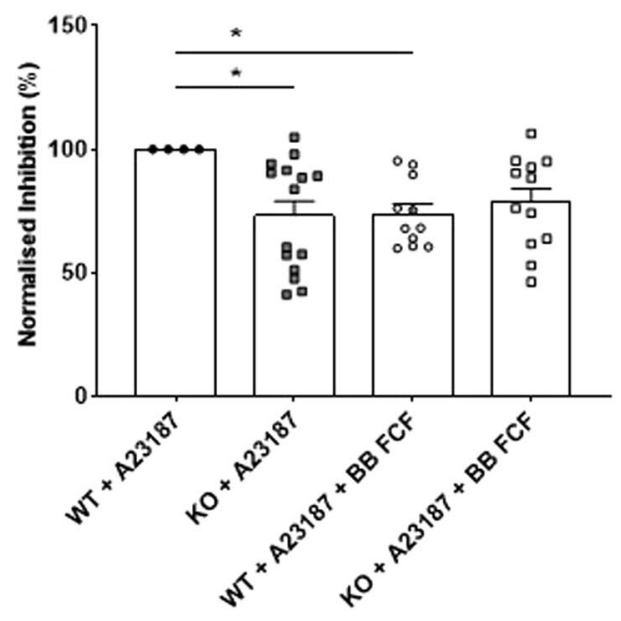

D

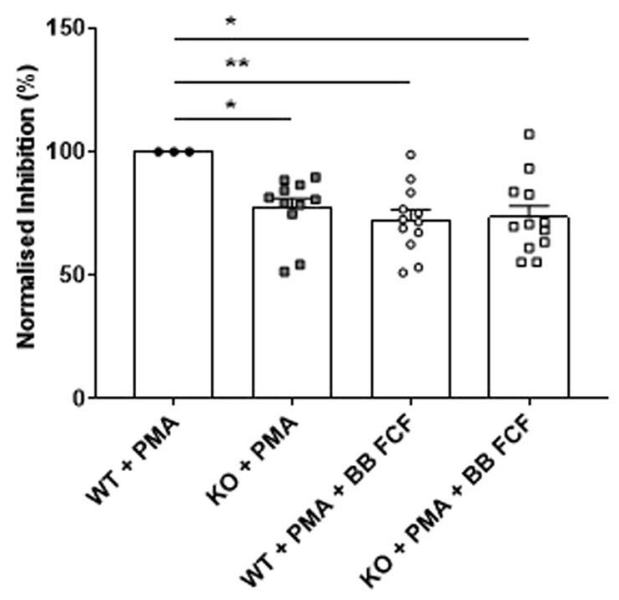

Figure 6. Chemical inhibition of Panx1 reduces NETosis in WT neutrophils. (A) Representative Kinetic of BB FCF dye $(1 \mu \mathrm{M})$ inhibition of NETosis in WT neutrophils with A23187 stimulation. (B) Quantification of fluorescence measurements at $6 \mathrm{~h}(\mathrm{~N}=4, \mathrm{n}=7)$. (C) Representative Kinetic of BB-FCF dye $(1 \mu \mathrm{M})$ inhibition of NETosis in WT neutrophils with PMA stimulation. (D) Quantification of fluorescence measurements at $6 \mathrm{~h}$ $(\mathrm{N}=3, \mathrm{n}=6)^{*} \mathrm{P}<0.05 ;{ }^{* *} \mathrm{P}<0.01$.

NETosis, including rise in intracellular calcium and cell swelling ${ }^{13,21}$, are also known triggers of Panx1 channel opening ${ }^{33,34}$. Thus, we propose that reduced ATP released by Pan $x 1^{-1-}$ BMDNs may lower the ability of the nucleotide and its metabolites to activate purinergic receptors, thus increasing the delay before the point of no return. Once this point is reached and NETosis occurs in some BMDNs, ATP will be released due to membrane rupture, thus accelerating NETosis in the surrounding neutrophils by activating the purinergic signalling cascade. Importantly, we show here that pharmacological inhibition of Panx1 channels with BB-FCF is sufficient to decrease the number of neutrophils undergoing NETosis in response to A23187 and PMA.

The identification of the molecular pathways that contribute to chromatin swelling are relevant to identify therapeutic targets. BB-FCF is an FDA-approved blue dye used for food colouring and, possibly in combination with other purinergic receptor inhibitors, could represent an efficient mean to reduce detrimental NETosis, particularly under excessive inflammatory conditions. This is not only of relevance for (auto-) inflammatory diseases but also for cancer as NETs induced by inflammation could promote the awakening of dormant cancer cells $\mathrm{s}^{35}$. Future work on animal models whereby Panxl is selectively deleted from neutrophils will shed some light on the rational of targeting this channel in inflamed tissues.

\section{Materials and Methods}

Mice. The Swiss Federal veterinary authorities ("Autorité Cantonale, Direction Générale de la Santé) approved all animal experimentation in accordance to established guidelines and regulations. NETosis experiments were performed using WT and Panx $1^{-1-}$ mice on a C57BL/6 background. Pan $x 1^{-1-}$ mice were previously characterised $^{36}$. All experiments were performed using sex and age-matched animals. Mice were bred in the in-house animal facility and kept under standard housing conditions with fixed light/dark cycle. 
Neutrophil isolation. Neutrophils were isolated from bone marrow. Mice were killed, femurs and tibiae of the lower limbs were exposed and flushed to obtain bone marrow in calcium free PBS with EDTA (1 mM, Promega, \#V4231) and fetal bovine serum (2\%, ThermoFisher Scientific, \#10270098). Neutrophil isolation and purification were carried out using EasySep ${ }^{\mathrm{TM}}$ negative selection mouse neutrophil enrichment kit and magnet, according to manufacturer's protocol (Stemcell Technologies, \#19762, \#18000). Bone marrow derived neutrophils (BMDNs) were washed and maintained in RPMI 1640 without phenol red (ThermoFisher Scientific, \#11835-063) supplemented with HEPES (10 mM, ThermoFisher Scientific, \#15630056) prior to immediate use.

Real-time PCR. BMDN RNA extraction was done using RNeasy mini kit (Qiagen, \#74106). Genomic DNA removal and cDNA synthesis were performed using the RT Quantitect kit (Qiagen, \#205311) with the UNOII PCR thermocycler (Biometra GmbH, Göttingen, Germany). qPCR was performed using TAQMAN gene primers (ThermoFisher Scientific, \#Mm00450900_m1, \#Mm01308054_m1, \#Mm00552586_m1 and \#Mm01179639_s1 for Panx1, Panx2, Panx3 and Cx43, respectively) with the Applied Biosystems StepOne Plus ${ }^{\mathrm{TM}}$ Real Time PCR system (ThermoFisher Scientific), according to the manufacturer's pre-set amplification protocol. Gene expression was normalised to mouse glyceraldehyde 3-phosphate dehydrogenase (GAPDH) expression. Values were expressed as $2^{-\triangle C T}$, according to the procedure described by Schmittgen and Lival, $2008^{37}$. Transcript detection with CT values above 34 were considered as undetected.

Immunofluorescence. $\quad 10^{5} \mathrm{BMDNs}$ were seeded on coverslips by centrifugation in 24-well plate and were fixed with paraformaldehyde (4\%) for $20 \mathrm{~min}$ at room temperature. Next, permeabilisation was performed with Triton-X100 (0.3\%), neutralisation with ammonium chloride $(0.5 \mathrm{M})$, and blocking with PBS containing bovine serum albumin (BSA; $\%$ ). The chicken anti-mouse Panx1 (1:500), recognizing amino acids $414-425^{38}$, citrullinated histone 3 (1:300, Abcam, AB5103) and neutrophil marker NIMP-R14 (1/250, Santa Cruz, sc-59338) primary antibodies were then applied overnight. Goat anti-chicken (1:500) DyLight488 antibody (Jackson Laboratories), goat anti-rabbit (1:2000) Alexa 488 (Thermofisher Scientific) and IgG anti-rat (1:100) rhodamine secondary antibodies were then applied for $2 \mathrm{~h}$ at room temperature (RT) with DAPI counterstaining. Samples were mounted on coverslips with Vectashield (Vector Laboratories, \#H-1000). Images were acquired using inverted Zeiss LSM700 laser-scanning confocal microscope (Carl Zeiss) through 10x, 20x, 40x or 63x oil immersion objectives. Quantification was performed using Fiji software. Histone $\mathrm{H} 3$ citrullination and DAPI fluorescence were quantified as area per image, while Panxl fluorescence was quantified as integrated density per region of interest (ROI) in WT and Panx $1^{-1-}$ BMDNs. Ratio values were expressed as arbitrary unit.

Western blot. BMDNs and splenocytes from WT and Panx $1^{-1-}$ mice were incubated in RIPA lysis buffer (1 mM PMSF, $0.2 \%$ Na-deoxycholate, $2 \mathrm{mM}$ Na-orthovanadate, $10 \mathrm{mM} \mathrm{NaF}, 1 \mathrm{X}$ Roche Protease Inhibitor, $0.05 \%$ SDS, 1 X NP40, $1 \mathrm{mM}$ EDTA, pH 7.4.) for 15 minutes on ice. Samples were then centrifuged at $10^{\prime} 000 \mathrm{~g}$ and $4^{\circ} \mathrm{C}$ for 15 minutes. Proteins were quantified by Pierce bicinchoninic acid (BCA) assay (ThermoFisher Scientific). $50 \mu \mathrm{g}$ and $10 \mu \mathrm{g}$ of total protein from BMDNs and splenocytes, respectively, were separated on a sodium dodecyl sulphate (SDS) polyacrylamide gel and transferred on nitrocellulose membrane. Membrane was blocked in PBS-Tween (0.01\%-PBST) buffer containing 5\% defatted milk for 1 hour at RT with agitation. Monoclonal Panx 1 (D9M1C Cell Signalling, 91137) antibodies were diluted 1: 1000 in 5\% milk in PBST for overnight incubation at $4{ }^{\circ} \mathrm{C}$, followed by 2 hours incubation at RT with goat anti-rabbit horse radish peroxidase (HRP)-conjugated antibody (Jackson ImmunoResearch Laboratories). Revelation was performed using WesternBright ${ }^{\mathrm{TM}}$ Sirius $^{\mathrm{TM}}$ (Advansta). Total amount of loaded protein was verified using Ponceau red staining.

Panx1 functional assay. For ATP release assay, BMDNs were left to equilibrate in Tyrode's buffer (124 mM $\mathrm{NaCl}, 2.4 \mathrm{mM} \mathrm{KCl}, 10.8 \mathrm{mM} \mathrm{NaHCO}_{3}, 0.4 \mathrm{mM} \mathrm{NaH}_{2} \mathrm{PO}_{4} * \mathrm{H}_{2} \mathrm{O}, 0.9 \mathrm{mM} \mathrm{MgCl}_{2} * 6 \mathrm{H}_{2} \mathrm{O}, 1.8 \mathrm{mM} \mathrm{CaCl}_{2} * 6 \mathrm{H}_{2} \mathrm{O}$, $\mathrm{pH}$ 7.35). Panx 1 channel mediated ATP release was activated by reducing osmolarity of the Tyrode's buffer by decreasing $\mathrm{NaCl}$ to $30.24 \mathrm{mM}$. WT and Panx1 $^{-1-}$ BMDNs were stimulated with appropriate controls for $5 \mathrm{~min}$. Cells were centrifuged at $1200 \mathrm{rpm}$ for 5 minutes and supernatants were collected for ATP measurement using the ATP bioluminescent assay kit (\#FLAA, Sigma Aldrich), according to the manufacturer's instructions.

For dye uptake assay, BMDNs were seeded in 384-well plate and maintained in a physiological solution (136 mM NaCl, $4 \mathrm{mM} \mathrm{KCl,} 1 \mathrm{mM} \mathrm{CaCl}_{2}, 1 \mathrm{mM} \mathrm{MgCl}_{2}$, and $2.5 \mathrm{mM}$ glucose, buffered to $\mathrm{pH} 7.4$ with $10 \mathrm{mM}$ HEPES-NaOH solution). YO-PRO-1 Iodide ( $5 \mu \mathrm{M}$, ThermoFisher Scientific, \#Y3603) was added to equilibrate cells. Hypo-osmotic shock was induced to maximally open Panx1 channels. Inhibitors of Panx1 BB-FCF dye-Erioglaucine disodium salt (Sigma Aldrich, \#80717) ${ }^{24,25}$ and spironolactone (Sigma Aldrich, \#S3378) ${ }^{39}$ were prepared in DMSO and used at $1 \mu \mathrm{M}$ and $100 \mu \mathrm{M}$, respectively. Global quantification of YO-PRO-1 dye uptake per well was performed using the FDSS/ $\mu$ CELL Functional Drug Screening System (Hamamatsu Photonics).

In Vitro quantification of NET formation. NETosis measurements were made as follows; $10^{5} \mathrm{BMDNs}$ were seeded in 96-well assay plate (Corning \#3603, Sigma Aldrich). 50 nM PMA or $1 \mu$ M A23187 (Sigma Aldrich, \#P1585, \#C7522 respectively) were added to wells in duplicates/triplicates with DMSO control included. The cell impermeable SYTOX green nucleic acid stain $(5 \mu \mathrm{M}$, ThermoFisher Scientific \#S7020) was added. SYTOX green stain measurement was performed using Spark Microplate Reader (Tecan) fitted with monochromatic filter 504/523 nm. $1 \mu \mathrm{M}$ BB-FCF was used for Panx1 inhibition with appropriate controls. ATP scavenger apyrase grade VII (50 U/ml, Sigma Aldrich, \#A6535), P2Y 2 receptor antagonist AR-C 118925XX (500 nM, Tocris, \#4890) and CD39 ARL 67156 trisodium salt $(100 \mu \mathrm{M}$, Tocris, \#1283) were used for the purinergic signalling experiments involving ATP release. $10 \mathrm{mM}$ N-acetyl cysteine (NAC, Sigma Aldrich \#A0737) was used for ROS inhibition. For time-lapse imaging, SYTOX green dye $(5 \mu \mathrm{M})$ was added to BMDNs seeded in 96-well assay plate. Cells were stimulated with $1 \mu \mathrm{M}$ A23187 for $30 \mathrm{~min}$ in the presence of appropriate controls. Images were acquired at 
10 minutes intervals for $5 \mathrm{~h}$ using the ImageXpress Micro Widefield High Content Screening System (Molecular Devices, San Jose, USA). MetaXpress 2.0 software was used to reconstitute images for video analysis.

Statistics. Statistical analyses were performed using Prism 8.0.2 (Graph Pad Prism). Data was expressed as mean \pm SEM. Nonlinear regression analysis was performed using the log (agonist) vs. response- Variable slope (four parameters) for SYTOX fluorescence traces. Background fluorescence was subtracted and the maximal fluorescence value (MAX), Hill slope and the time (ET50) for response halfway between the basal (Min) value and the maximal (Max) value were determined. One-way ANOVA and non-parametric Student $t$ test (Mann-Whitney) were used when appropriate and $* \mathrm{P} \leq 0.05, * * \mathrm{P} \leq 0.01, * * * \mathrm{P} \leq 0.001$, $* * * * \mathrm{P} \leq 0.0001$ were considered significant.

Received: 23 May 2019; Accepted: 24 October 2019;

Published online: 12 November 2019

\section{References}

1. Vong, L., Sherman, P. M. \& Glogauer, M. Quantification and visualization of neutrophil extracellular traps (NETs) from murine bone marrow-derived neutrophils. Methods Mol Biol 1031, 41-50, https://doi.org/10.1007/978-1-62703-481-4_5 (2013).

2. Brinkmann, V. et al. Neutrophil extracellular traps kill bacteria. Science 303, 1532-1535, https://doi.org/10.1126/science.1092385 (2004).

3. Delgado-Rizo, V. et al. Neutrophil Extracellular Traps and Its Implications in Inflammation: An Overview. Front Immunol 8, 81, https://doi.org/10.3389/fimmu.2017.00081 (2017).

4. Yipp, B. G. et al. Infection-induced NETosis is a dynamic process involving neutrophil multitasking in vivo. Nat Med 18, 1386-1393, https://doi.org/10.1038/nm.2847 (2012).

5. Berthelot, J. M., Le Goff, B., Neel, A., Maugars, Y. \& Hamidou, M. NETosis: At the crossroads of rheumatoid arthritis, lupus, and vasculitis. Joint Bone Spine 84, 255-262, https://doi.org/10.1016/j.jbspin.2016.05.013 (2017).

6. Takei, H., Araki, A., Watanabe, H., Ichinose, A. \& Sendo, F. Rapid killing of human neutrophils by the potent activator phorbol 12-myristate 13-acetate (PMA) accompanied by changes different from typical apoptosis or necrosis. J Leukoc Biol 59, 229-240 (1996).

7. Remijsen, Q. et al. Neutrophil extracellular trap cell death requires both autophagy and superoxide generation. Cell Res 21, 290-304, https://doi.org/10.1038/cr.2010.150 (2011).

8. Yipp, B. G. \& Kubes, P. NETosis: how vital is it? Blood 122, 2784-2794, https://doi.org/10.1182/blood-2013-04-457671 (2013).

9. Douda, D. N., Khan, M. A., Grasemann, H. \& Palaniyar, N. SK3 channel and mitochondrial ROS mediate NADPH oxidaseindependent NETosis induced by calcium influx. Proc Natl Acad Sci USA 112, 2817-2822, https://doi.org/10.1073/pnas.1414055112 (2015).

10. Yousefi, S., Mihalache, C., Kozlowski, E., Schmid, I. \& Simon, H. U. Viable neutrophils release mitochondrial DNA to form neutrophil extracellular traps. Cell Death Differ 16, 1438-1444, https://doi.org/10.1038/cdd.2009.96 (2009).

11. Konig, M. F. \& Andrade, F. A Critical Reappraisal of Neutrophil Extracellular Traps and NETosis Mimics Based on Differential Requirements for Protein Citrullination. Front Immunol 7, 461, https://doi.org/10.3389/fimmu.2016.00461 (2016)

12. Kenny, E. F. et al. Diverse stimuli engage different neutrophil extracellular trap pathways. Elife 6, https://doi.org/10.7554/eLife.24437 (2017).

13. Neubert, E. et al. Chromatin swelling drives neutrophil extracellular trap release. Nat Commun 9, 3767, https://doi.org/10.1038/ s41467-018-06263-5 (2018).

14. Pieterse, E., Rother, N., Yanginlar, C., Hilbrands, L. B. \& van der Vlag, J. Neutrophils Discriminate between Lipopolysaccharides of Different Bacterial Sources and Selectively Release Neutrophil Extracellular Traps. Front Immunol 7, 484, https://doi.org/10.3389/ fimmu.2016.00484 (2016)

15. Gray, R. D. et al. Delayed neutrophil apoptosis enhances NET formation in cystic fibrosis. Thorax 73, 134-144, https://doi. org/10.1136/thoraxjnl-2017-210134 (2018).

16. D'Hondt, C. et al. Pannexin channels in ATP release and beyond: an unexpected rendezvous at the endoplasmic reticulum. Cell Signal 23, 305-316, https://doi.org/10.1016/j.cellsig.2010.07.018 (2011).

17. Boassa, D. et al. Pannexin 1 channels contain a glycosylation site that targets the hexamer to the plasma membrane. J Biol Chem 282, 31733-31743, https://doi.org/10.1074/jbc.M702422200 (2007).

18. Penuela, S., Gehi, R. \& Laird, D. W. The biochemistry and function of pannexin channels. Biochim Biophys Acta 1828, 15-22, https:// doi.org/10.1016/j.bbamem.2012.01.017 (2013).

19. Chen, Y. et al. Purinergic signaling: a fundamental mechanism in neutrophil activation. Sci Signal 3, ra45, https://doi.org/10.1126/ scisignal.2000549(2010).

20. Wang, X. \& Chen, D. Purinergic Regulation of Neutrophil Function. Front Immunol 9, 399, https://doi.org/10.3389/ fimmu.2018.00399 (2018).

21. de Bont, C. M., Koopman, W. J. H., Boelens, W. C. \& Pruijn, G. J. M. Stimulus-dependent chromatin dynamics, citrullination, calcium signalling and ROS production during NET formation. Biochim Biophys Acta Mol Cell Res 1865, 1621-1629, https://doi. org/10.1016/j.bbamcr.2018.08.014 (2018).

22. Abitbol, J. M. et al. Double deletion of Panxl and Panx3 affects skin and bone but not hearing. J Mol Med 97, 723-736, https://doi. org/10.1007/s00109-019-01779-9 (2019).

23. Wang, N. et al. Paracrine signaling through plasma membrane hemichannels. Biochim Biophys Acta 1828, 35-50, https://doi. org/10.1016/j.bbamem.2012.07.002 (2013).

24. Wang, J., Jackson, D. G. \& Dahl, G. The food dye FD\&C Blue No. 1 is a selective inhibitor of the ATP release channel Panx1. J Gen Physiol 141, 649-656, https://doi.org/10.1085/jgp.201310966 (2013).

25. Molica, F., Nolli, S., Fontana, P. \& Kwak, B. R. Turbidimetry on Human Washed Platelets: The Effect of the Pannexin1-inhibitor Brilliant Blue FCF on Collagen-induced Aggregation. J Vis Exp 2017(122), 55525, https://doi.org/10.3791/55525 (2017).

26. Chen, Y. et al. Inhibition of Neutrophils by Hypertonic Saline Involves Pannexin-1, CD39, CD73, and Other Ectonucleotidases. Shock 44, 221-227, https://doi.org/10.1097/SHK.0000000000000402 (2015).

27. Barletta, K. E., Ley, K. \& Mehrad, B. Regulation of neutrophil function by adenosine. Arterioscler Thromb Vasc Biol 32, 856-864, https://doi.org/10.1161/ATVBAHA.111.226845 (2012).

28. Villagra-Blanco, R. et al. Bovine Polymorphonuclear Neutrophils Cast Neutrophil Extracellular Traps against the Abortive Parasite Neospora caninum. Front Immunol 8, 606, https://doi.org/10.3389/fimmu.2017.00606 (2017).

29. Sil, P. et al. P2Y6 Receptor Antagonist MRS2578 Inhibits Neutrophil Activation and Aggregated Neutrophil Extracellular Trap Formation Induced by Gout-Associated Monosodium Urate Crystals. J Immunol 198, 428-442, https://doi.org/10.4049/ jimmunol.1600766 (2017).

30. Esseltine, J. L. \& Laird, D. W. Next-Generation Connexin and Pannexin Cell Biology. Trends Cell Biol 26, 944-955, https://doi. org/10.1016/j.tcb.2016.06.003 (2016). 
31. Chen, Y., Hashiguchi, N., Yip, L. \& Junger, W. G. Hypertonic saline enhances neutrophil elastase release through activation of P2 and A3 receptors. Am J Physiol Cell Physiol 290, C1051-1059, https://doi.org/10.1152/ajpcell.00216.2005 (2006).

32. Bao, Y., Chen, Y., Ledderose, C., Li, L. \& Junger, W. G. Pannexin 1 channels link chemoattractant receptor signaling to local excitation and global inhibition responses at the front and back of polarized neutrophils. J Biol Chem 288, 22650-22657, https://doi. org/10.1074/jbc.M113.476283 (2013).

33. Lohman, A. W. \& Isakson, B. E. Differentiating connexin hemichannels and pannexin channels in cellular ATP release. FEBS Lett 588, 1379-1388, https://doi.org/10.1016/j.febslet.2014.02.004 (2014).

34. Chiu, Y. H., Schappe, M. S., Desai, B. N. \& Bayliss, D. A. Revisiting multimodal activation and channel properties of Pannexin 1.J Gen Physiol 150, 19-39, https://doi.org/10.1085/jgp.201711888 (2018).

35. Albrengues, J. et al. Neutrophil extracellular traps produced during inflammation awaken dormant cancer cells in mice. Science 361, https://doi.org/10.1126/science.aao4227 (2018).

36. Anselmi, F. et al. ATP release through connexin hemichannels and gap junction transfer of second messengers propagate Ca2+ signals across the inner ear. Proc Natl Acad Sci USA 105, 18770-18775, https://doi.org/10.1073/pnas.0800793105 (2008).

37. Schmittgen, T. D. \& Livak, K. J. Analyzing real-time PCR data by the comparative C(T) method. Nat Protoc 3, 1101-1108 (2008).

38. Molica, F. et al. Functional role of a polymorphism in the Pannexin1 gene in collagen-induced platelet aggregation. Thromb Haemost 114, 325-336, https://doi.org/10.1160/TH14-11-0981 (2015).

39. Good, M. E. et al. Pannexin 1 Channels as an Unexpected New Target of the Anti-Hypertensive Drug Spironolactone. Circ Res 122, 606-615, https://doi.org/10.1161/Circresaha.117.312380 (2018).

\section{Acknowledgements}

This work was supported by the Swiss National Science Foundation (\#310030_172909/1 to MC and \#310030_182573 to BRK). We would like to thank the University of Geneva Faculty of Medicine Core FacilitiesFlow Cytometry, Bioimaging and READS unit for excellent technical support as well as Tecan Switzerland for lending the Spark microplate reader. We also thank Dr. Filippo Molica and Dr. Juliette Simonin for scientific input and Bernard Foglia for maintaining the mouse lines used in this study.

\section{Author contributions}

M.C. and A.S. designed research, A.S. performed experiments, M.Bac. assisted with animal experimentation, M.Bad. performed Western blots. A.S. and M.C. analysed data. A.S., B.R.K. and M.C. wrote manuscript. All authors reviewed the manuscript.

\section{Competing interests}

The authors declare no competing interests.

\section{Additional information}

Supplementary information is available for this paper at https://doi.org/10.1038/s41598-019-53058-9.

Correspondence and requests for materials should be addressed to M.C.

Reprints and permissions information is available at www.nature.com/reprints.

Publisher's note Springer Nature remains neutral with regard to jurisdictional claims in published maps and institutional affiliations.

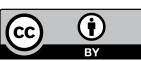

Open Access This article is licensed under a Creative Commons Attribution 4.0 International License, which permits use, sharing, adaptation, distribution and reproduction in any medium or format, as long as you give appropriate credit to the original author(s) and the source, provide a link to the Creative Commons license, and indicate if changes were made. The images or other third party material in this article are included in the article's Creative Commons license, unless indicated otherwise in a credit line to the material. If material is not included in the article's Creative Commons license and your intended use is not permitted by statutory regulation or exceeds the permitted use, you will need to obtain permission directly from the copyright holder. To view a copy of this license, visit http://creativecommons.org/licenses/by/4.0/.

(C) The Author(s) 2019 